\section{Intramodal and crossmodal sensory transfer of visual and auditory temporal patterns*}

\section{LEO RUBINSTEIN** and ERNEST M. GRUENBERG $\dagger$ \\ Columbia University, New York, New York}

Temporal pattern perception for vision and audition was measured using pair comparisons of precisely determined rhythms as test items. Visual rhythms were more difficult to match than were comparable auditory ones. Crossmodal transfer equaled intramodal transfer in one experiment but was inferior in another. The differences between intra- and crossmodal transfer were related to the frequency of pattern elements, with crossmodal performance decreasing more when frequency rose. The nine test items showed a consistency in relative difficulty across experimental conditions. Differences in item difficulty were related to the symmetry of the first pattern in the item.

Two types of sensory transfer can be defined: Intersensory, or crossmodal, transfer occurs when an organism uses information from one sense mode to perform a task or solve a problem involving a different sense mode; intrasensory, or intramodal, transfer occurs when both the information and the task are in the same sense mode. Example: (1) A mechanic is looking through his tools for a wrench to fit a nut he sees; (2) there is a blackout and he now must feel both the nut and among the tools; (3) he finds his flashlight, points it at the nut while fumbling with the tools; (4) he shifts tactics and points the flashlight in the tool bag and holds the nut with the other hand. These four problems illustrate successively visual-visual, haptic-haptic, visual-haptic, and haptic-visual sensory transfer. The first two are intramodal and the latter two are crossmodal.

The mechanic's problem illustrates the simplest level of sensory transfer, matching of equivalent stimuli (Ettlinger, 1967). To find the wrench that fits the nut, he may engage in two comparison processes: (1) Pair comparison-a standard, the nut, is paired with a comparison, the wrench; if they match, he says "same," if not, he says "different." (2) "Matching to sample"-a standard, the nut, is contrasted to a set of two or more alternatives, the wrenches; he picks the alternative that corresponds most to the standard. Research studies differ from this everyday situation in that the

*This research was supported with funds from NIMH Grants MH 08609-05 and MH 08609-06.

* * Requests for reprints should be addressed to Dr, Leo Rubinstein, Psychiatric Epidemiology Research Unit, 722 West 168 th Street, New York, New York 10032.

t The authors wish to thank Mr. M. Tobin of the New York State Psychiatric Institute, for the design and assembly of the electronic system used to present the patterns, and Dr.J. Rutschmann, for the use of the electronic system used to produce visual stimuli. items to be matched are varied systematically.

The present experiments are concerned with comparing crossmodal matching between vision and hearing to intramodal matching within these sense modes in normal adults.

Previous studies of crossmodal matching between vision and hearing used some version or modification of the Birch and Belmont test (1964). It is an audiovisual "matching to sample" task in which the S listens to a pattern being tapped out, then selects from among three or four Morse-code-like patterns pictured on a card the one which corresponds to what he heard. The differences obtained between groups on audiovisual matching ability, using this test, may be affected by three other types of performance implicit in the test structure: (1) spatiotemporal transformation-a temporal auditory pattern is compared to spatial visual patterns; (2) counting-in some items the comparison pattems do not all contain the same number of elements; (3) length perception-in other items the visual comparison patterns do not have the same extent. Although these performances might not differ as much in normal adults as they do among the groups to whom the Birch and Belmont test has been given most often-children and the brain damaged-they should be under experimental control.

These earlier studies, while evaluating audiovisual transfer, did not report on either the conıplementary crossmodal performance, viz, visuoauditory transfer, or intramodal transfer for both vision and hearing. The inferiority of crossmudal transfer reported by Birch and Lefford (1963) can be due to comparing it to the intramodal transfer within the better of the two sense modes in question; on the other hand, comparing crossmodal transfer to the intlamodal transfer within the worst of the two senses should result in crossmodal superiority (Bryant, 1968). To make a valid comparison between intramodal and crossmodal transfer, one must include all intramodal and crossmodal combinations in the test. Muehl and Kremenak (1966) introduced the necessary intramodal controls, added the complementary visuoauditory task, and changed the test from "matching to sample" to "same-different." Their items, however, were different among the four tasks, and differences in item difficulty could have affected the differences obtained among their conditions; also, their crossmodal tests still involved spatiotemporal comparisons from vision to hearing, while the intramodal tests for vision and hearing involved only spatial and temporal comparisons, respectively.

\section{METHOD}

The present two experiments were designed to yield highly comparable crossmodal and intramodal tests. All patterns were temporal, contained the same number of elements (three intervals demarcated by four stimuli), and were of the same duration within an experiment. The three intervals consisted of two short and one long interval, all of whose values remained constant within an experiment, as did the physical aspects of the stimuli. Thus, the only relevant aspect for identifying a pattern was the arrangement of the intervals, i.e., the position of the long interval.

Since only three intervals comprised a pattern, the single long interval could occur between the first, middle, or final pair of stimuli. These three possibilities are identified, respectively, as Patterns 1, 2, and 3. They are diagramed in Fig. 1.

\section{Stimulation}

Binaural clicks were produced by applying a $1-\mathrm{msec}$ square pulse of $3 \mathrm{~V}$, taken from the logic of the programming unit, to a set of Sony DR-3C earphones. The intensity was approximately $70 \mathrm{~dB}$ above E's sensation level and was clearly audible and comfortable to listen to.

Light flashes were produced by

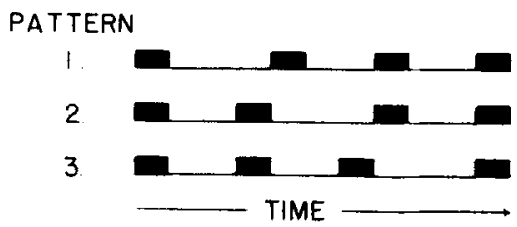

Fig. 1. Three temporal patterns; black rectangles represent stimuli. Small and large gaps between stimuli represent "short" and "long" intervals, respectively. Relative durations of stimuli and intervals are not drawn to scale. 
triggering a Sylvania R1131C glow modulator tube electronically. (A complete description of the triggering, gating, and Calibration of this system is given in Rubinstein, 1964.) In order to minimize the variability in the rise-decay time of the glow modulator, the ions in the tube were kept at an excited level by the ultraviolet radiation from an argon lamp. The duration of each flash was $3 \pm .1 \mathrm{msec}$. To the $S$, the flash appeared to come from a circular test patch of ground glass, $.5 \mathrm{~cm}$ in diam, within a black ring, $3 \mathrm{~cm}$ in diam, itself surrounded by gray cardboard. This viewing area was contained in a box and illuminated by a 7.5.W incandescent bulb which was out of the S's view. All luminances were measured with a Macbeth illuminometer placed 3 in. from the source and were as follows: test patch, $2.7 \log \mathrm{mL}$; black ring, $.4 \mathrm{~mL}$; gray surround, $1.5 \mathrm{~mL}$.

$S$ viewed the test patch binocularly, his eyes 24.30 in. from the test patch, his head unrestrained. Such freedom of movement is not conventional in visual research; its existence, however, diminished the frequency of disruptive afterimages and could permit eventual comparison of the present data with those of hyperkinetic groups, such as some young children or some schizophrenics, both of whom are difficult to test under any conditions and whose emotional response to confinement could produce a confounding variable.

The testing room was an office illuminated by two 40-W white fluorescent bulbs; the room was quiet except for occasional footsteps outside or above, which interrupted testing on one occasion.

\section{Timing}

All durations and intervals were produced by a computer-like assemblage of digital logic which received its program via punched paper tape. Since the computer operated two lines ahead of the tape reader (Teletype ASR 33), the variability in timing introduced by the mechanical aspects of the reader was not transmitted directly to the output devices, but was instead removed by momentary storage in the computer, leaving only the program information, which was emitted with the precision of the computer's own clock; the imprecision of this clock was less than $1 \%$ of the programmed values. The durations of the long and short intervals in Experiment I (fast) and Experiment II (slow) are shown in Table 1. For example, the table shows that in the slow experiment the "long" interval in the visual patterns was $.487 \mathrm{sec}, 45 \%$ longer than the "short" interval in that experiment.

The values of the long interval were greater for visual than for auditory patterns because our own pilot work, as well as the results of other studies, indicated that, for patterns having identical temporal constants, visual patterns were more difficult to recognize than were auditory patterns (Cole et al, 1961; Fraisse, 1948; Gardner, 1968; Garner \& Gottwald, 1968; Gault \& Goodfellow, 1938).

It was expected that the increased duration of the long interval in the visual patterns would make them easier to discriminate and thus equal or surpass the recognizability of the comparable auditory patterns.

The absolute durations of the intervals in Experiment II were increased beyond those in Experiment I because, in Experiment I, some Ss saw three flashes instead of four in the visual patterns. This phenomenon is common at interflash intervals of $.222 \mathrm{sec}$ but rarer at interflash intervals of .33 sec (Taubman, 1950; Thor $\&$ Holden, 1970). The relative durations of the intervals in Experiment II were raised as well to (1) increase the percentage of correct responses and (2) to equate the recognizability of the auditory and visual patterns. The values selected were based on pilot experiments with the present system.

\section{Procedure}

Pilot work using "matching to sample" indicated that the time required to present the alternative patterns might cause memory to influence the likelihood of correct recognition, thus possibly confounding the results. Therefore, the matching task used was "same-different," which minimized the influence of memory by including only two patterns per test item. Eliminating the role of memory entirely by using simultaneous rather than successive presentation of the patterns would change the task from one of recognizing patterns to one of detecting asynchrony, a different task indeed.

Only nine pair comparisons between three patterns are possible; three of these comparisons, or items, 1-1, 2-2, and 3-3, require a "same" response; the remaining six, $1-2,1-3,2-1,2-3,3-1$, and $3-2$, require a "different" response. To offset being outnumbered $2: 1$, the "same" items were presented twice as often. Items were arranged in an order that met three restrictions: (1) Half the "same" items were preceded by "same" items and half were preceded by "different" items; (2) three or more consecutive occurrences of either "same" or "different" items were prohibited; (3) in each successive group of six items (12 patterns), each pattern occurred four times, thus assuring an even distribution of occurrence of the patterns over time.
Table 1

Experimental Conditions

\begin{tabular}{lcccc}
\hline & \multicolumn{2}{c}{ Absolute } & & \multicolumn{2}{c}{ Relative } \\
\cline { 2 - 3 } Inter- & Auditory & Visual & Auditory & Visual \\
\hline & \multicolumn{3}{c}{ Experiment I ("Fast") } \\
Short & .222 & .222 & 1.00 & 1.00 \\
Long & .282 & .304 & 1.28 & 1.38 \\
& Experiment II ("Slow") & \\
Short & .333 & .333 & 1.00 & 1.00 \\
Long & .444 & .487 & 1.33 & 1.45 \\
\hline
\end{tabular}

Note-Durations of short and long intervals in visual and auditory patterns; absolute values in seconds; relative values calculated setting "short" interval for each experiment at 1.0 .

To make the proper intramodal and crossmodal comparisons, four modal combinations were necessary: auditory-auditory (AA), visual-visual (VV), auditory-visual (AV), and visual-auditory (VA). Each modal combination contained the same items but ordered differently, subject to the restrictions above. The order of presentation of the two occasions of each of these combinations was AA, VV, $A V, V A, V A, A V, V V$, and AA. For half of the Ss the positions of $A V$ and $V A$ were reversed. The AA task was presented first because, being the most familiar, it would indicate rapidly whether $S$ would be able to perform the tasks with proficiency beyond chance.

Within each of the four modal combinations, each of the three "same" items occurred four times and each of the six "different" items occurred twice, making $4(3 \times 4+6 \times 2)=96$ items for a whole session. The 24 occurrences of each item were equally divided between the two occasions for each modal combination. Any single item consisted of a verbal "ready" signal followed $2-3 \mathrm{sec}$ later by the first pattern of the item, a 1-sec gap between the last element of the first pattern and the first element of the second pattern, the second pattern itself, and the interitem interval, during which the S's decision was manually recorded by $\mathrm{E}$, the investigator. The interitem interval was about $5 \mathrm{sec}$, depending on S's decision latency, and the sessjon lasted about $25 \mathrm{~min}$, with 1 -min rests at midsession and S's request.

The instructions to the Ss can be paraphrased as follows: I will ask you to compare rhythmic patterns of clicks or flashes. Each pattern will always have four elements. Tell me whether the second pattern has the same rhythm as the first. Half the patterns will be "same," half "different." If one pattern seems longer or shorter, then compare the relationships between the elements, i.e., a "cha-cha-cha" beat may be fast or slow, but it is still a "cha-cha-cha." 


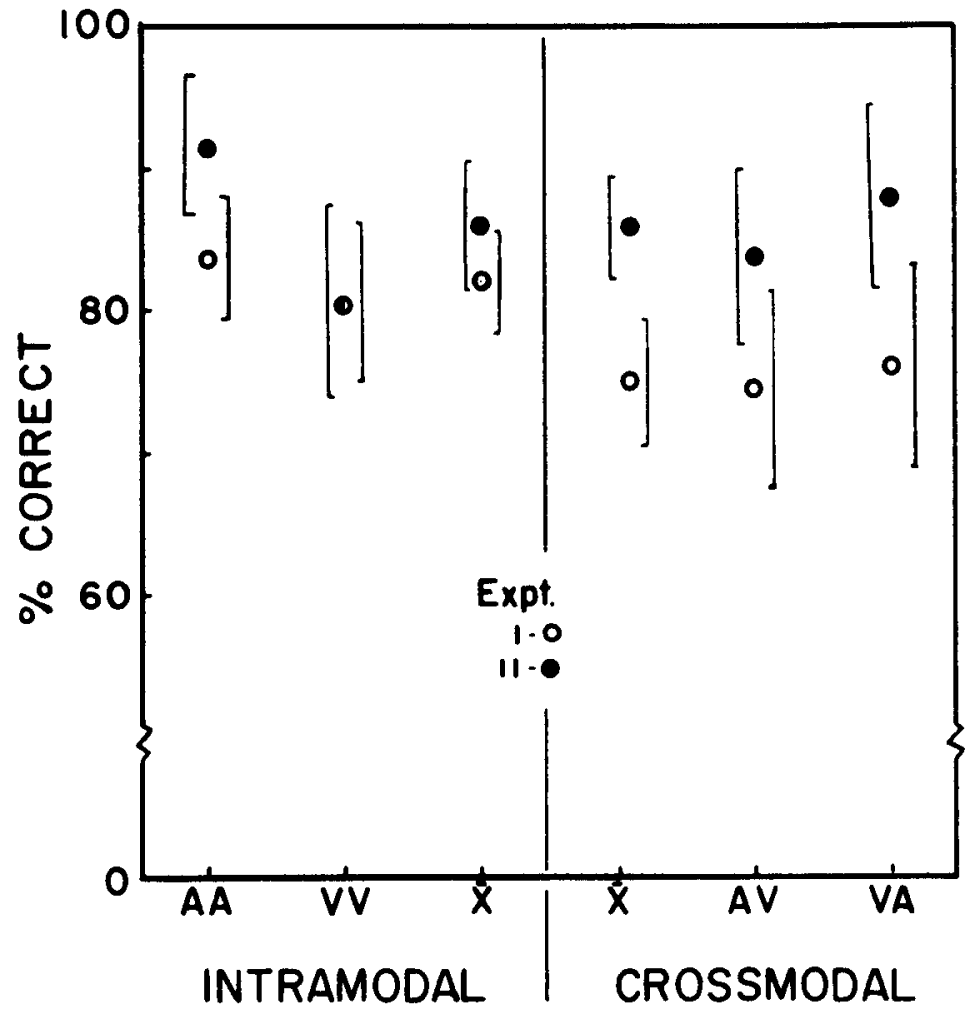

Fig. 2. Percentage of correct comparisons of temporal patterns for intramodal and crossmodal combinations of visual and auditory patterns. Brackets signify $\pm 2 \mathrm{SE}_{\mathbf{M}}$; $\overline{\mathrm{X}}=$ mean.

The instructions were followed by eight practice items, two from each modal combination, four being "same" and four "different." To clarify the task for $S$, he was told at once whether he was right or wrong on each of the practice items. No knowledge of results was provided for the test items themselves.

Ss were staff, student nurses, and graduate students, only the latter of whom were paid. All were between 20 and 40 years old. Of the 31 Ss, 13 were men and 17 were women; one man and one woman served in both experiments.

\section{Hypotheses}

It was expected that in each experiment: (1) The two intramodal tasks would yield equal scores, $\mathbf{A A}=\mathrm{VV}$. The visual patterns were designed to be as recognizable as the comparable auditory patterns by making the long interval relatively greater than the short intervals (see Table 1). (2) The two crossmodal tasks would yield equal scores, $A V=V A$. The order of presentation of sense modality within an item was not expected to be influential, and the $\mathrm{A}$ and $\mathrm{V}$ patterns were designed to be equally recognizable. (3) The combined intramodal scores would equal or surpass the combined crossmodal scores, $\mathrm{AA}+\mathrm{VV} \geqslant$ $A V+V A$. Superior or inferior crossmodal performance implies, respectively, stronger or weaker intersensory linkages than exist intrasensorily. Anatomical evidence points to the latter (Geschwind, 1965). (4) Items yield a smaller proportion of errors than would items beginning and ending with Patterns 1 and 3, "2"< $(" 1 "+" 3 ") / 2$. Pattern 2 is symmetrical, "good," and more recognizable (Ganer, 1970). (5) items beginning and ending with Patterns 1 and 3 would yield equal proportions of errors, "l" = "3." Patterns 1 and 3 are alike, except for being each other's reflection (Garner, 1970).

\section{RESULTS}

In Experiment $I$, three men and one woman did not complete testing because they perceived three flashes instead of four in the visual patterns. In Experiment II, two men and two women failed to achieve the criterion of performance on the VV task, so their results on all conditions were eliminated from analysis. The attrition of Ss left six men and six women in Experiment $I$ and live men and seven women in Experiment II. The performance criteria met by the $12 \mathrm{Ss}$ remaining in each experiment were seores on both the AA and $V V$ tasks that were not significantly below $83.3 \%$ correct. By having a criterion for intramodal performance below $100 \%$, it was possible for either AV or VA, or both, beginning and ending with Pattern 2 would to yield scores surpassing those on the particular intramodal task, AA or VV, which was more difficult. The designed equivalence of the intramodal tasks was no guarantee of their not differing in a small sample of Ss. With a $100 \%$ correct criterion, either crossmodal performance could at best only equal intramodal performance and would have a greater likelihood of falling short of intramodal performance, thus increasing the possibility of speciously proving the inferiority of crossmodal vs intramodal transfer.

All experimental hypotheses were tested by comparing the pairs of conditions concerned using $t$ tests of the differences between Ss. For each set of inferences (e.g., modal combinations, patterns), the .05 level of significance was selected, but, since several $t$ tests were performed on the same arrangement of data, the likelihood of obtaining a statistically significant $t$ was increased. To compensate for this, the significance level of .05 for each set was apportioned among the component tests, such that the greater the number of degrees of freedom in the test, the greater the significance level to be attained (Miller, 1966).

\section{Modal Combinations}

The relative frequency of correct responses, or ( lerror rate), for each modal combination is shown in Fig. 2.

In Experiment I the crossmodal tasks combined, $(\mathrm{AV}+\mathrm{VA}) / 2$, produced a significantly lower proportion of correct responses than did the intramodal tasks combined, $(\mathrm{AA}+\mathrm{VV}) / 2, .752$ vs .820 , respectively. Neither $A A$ and $V V$ nor $A V$ and VA were significantly different from each other. On the other hand, in Experiment II the crossmodal tasks combined were not significantly different from the intramodal tasks combined, the respective proportions of correct responses being .857 and .861 . Again AV did not differ from VA, but VV was significantly more difficult than AA, yielding a proportion correct of .805 vs .916 for AA. The one common result in the two experiments was that the two crossmodal tasks, AV and VA, did not differ from each other.

\section{Patterns}

The generality of relative item difficulty among four modal combinations in two experiments was tested by computing the Kendall coefficients of concordance, $W$, for these two sets of four rankings. W equaled .57 and .60 , which have a $\mathrm{p}$ of less than .02. The concordance of relative item difficulty among modal combinations permited the data from these combinations to be pooled for each $\mathrm{S}$ 


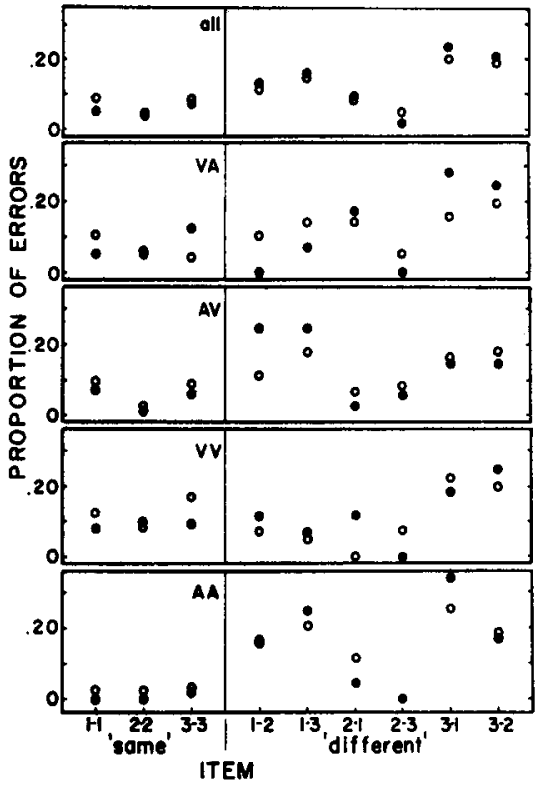

Fig. 3. Proportion of errors per item; the digit pair identifying each item refers to the patterns in the item as well as to the order in which the patterns occurred. "All" is the grand mean of the four modal combinations; $O$, Experiment $I ; \bullet$, Experiment II.

within each experiment. In both experiments the results with regard to pattern are quite similar. Across all modal combinations, the order of increasing difficulty of the nine items in Experiment I is: $2-3,2-2,2-1,3-3,1-1,1-2,1-3,3-2,3-1$. In Experiment II the order differs only in that Items 2.1 and 1-1 exchange ranks. The relative difficulty of the items is displayed in Fig. 3. The Spearman rank correlation coefficient, rho, of item difficulty between the two experiments is .93 . The rhos for item difficulty between the two experiments for each modal combination are: $\mathrm{AA}, .98 ; \mathrm{AV}, .91 ; \mathrm{VA}, .67 ; \mathrm{VV}, .51$. The .01 and .05 significance levels of tho for $\mathrm{N}=9$ are .78 and .60 , respectively. Table 2 shows the errors per item pooled across Ss and modalities.

In the analysis of items according to which pattern was first (row totals in Table 2), items beginning with Pattern 2 produced significantly fewer errors than the average of the items beginning with Patterns 1 and 3; the items beginning with Patterns 1 and 3 did not differ from each other. In the analysis of items according to which pattern was second (column totals in Table 2), no significant differences were found between the number of errors produced by Pattern 1 vs Pattern 3 or between the average of 1 and 3 vs Pattern 2.

Table 2

Number of Errors Per Item

\begin{tabular}{|c|c|c|c|c|c|}
\hline \multirow{2}{*}{$\begin{array}{l}\text { First } \\
\text { Pattern }\end{array}$} & \multicolumn{5}{|c|}{ Second Pattern } \\
\hline & 1 & 2 & 3 & Total & (\%) \\
\hline \multicolumn{6}{|c|}{ Experiment I ("Fast") } \\
\hline $\begin{array}{c}1 \\
2 \\
3 \\
\text { Total (\%) }\end{array}$ & $\begin{array}{l}36 \\
17 \\
39 \\
92(37)\end{array}$ & $\begin{array}{l}23 \\
19 \\
38 \\
80(33)\end{array}$ & $\begin{array}{l}30 \\
11 \\
33 \\
74 \quad(30)\end{array}$ & $\begin{array}{r}89 \\
47 \\
110 \\
246\end{array}$ & $\begin{array}{l}(36) \\
(19) \\
(45) \\
(100)\end{array}$ \\
\hline \multicolumn{6}{|c|}{ Experiment II ("Slow") } \\
\hline $\begin{array}{c}1 \\
2 \\
3 \\
\text { Total }(\%)\end{array}$ & $\begin{array}{l}16 \\
12 \\
30 \\
58(36)\end{array}$ & $\begin{array}{l}19 \\
13 \\
28 \\
60(37)\end{array}$ & $\begin{array}{r}21 \\
2 \\
21 \\
44 \quad(27)\end{array}$ & $\begin{array}{r}56 \\
27 \\
79 \\
162\end{array}$ & $\begin{array}{l}(35) \\
(17) \\
(49) \\
(100)\end{array}$ \\
\hline
\end{tabular}

Note-Each item consists of two patterns. To obtain errors for any item, enter row corresponding to first pattern in the item, enter column corresponding to second pattern in the item, e.g., Item 2-3 yielded $I 1$ and 2 errors in Experiments $I$ and $I I$, respectively.

\section{Modal Combinations}

\section{DISCUSSION}

In Experiments I and II, VV was equal to or more difficult than AA. When one considers that the ratio of the long to short interval was already $10 \%-12 \%$ greater in $\mathrm{VV}$, it is clear that temporal pattern perception is more difficult with visual than with comparable auditory stimuli. This is in accord with the findings of other investigators (Cole \& Chorover, 1961; Fraisse, 1948; Gardner, 1968; Garner \& Gottwald, 1968; Gault \& Goodfellow, 1938). Gault and Goodfellow observed that, for patterns with identical time relations, those containing auditory, visual, and tactile stimuli produced "correct" scores of $85 \%, 75 \%$, and $70 \%$, respectively, on a task similar to the present one. Moreover, 1,500 trials of practice produced improvement of only $2 \%-4 \%$ in performance and had little effect on the relative standing of the senses. Only an extrapolation from the data of White and Lichtenstein (1963) disagree with the present and previous findings. Their Fig. 3 indicates that, while audition is superior to vision in detecting syncopation of patterns having more than eight stimuli per second, vision surpasses audition for patterns having six to eight stimuli per second; the trends in their figures indicate that, for frequencies below six stimuli per second, vision would still surpass audition in rhythm perception.

Unfortunately, there is no earlier data on crossmodal perception of tenporal patterns in vision and audition, and the data obtained here are equivocal. In Experiment II, crossmodal transfer equals the average of the two intramodal conditions, which is just what would be predicted if one expected each sense mode to contribute equality to the crossmodal performance. In Experiment $\mathbf{l}$, however, crossmodal transfer is significantly poorer than intramodal transfer, and the mean crossmodal score is less than the lower intramodal score, i.e., VV. The major procedural difference between experiments was the duration of the interstimulus intervals; the patterns in Experiment $I$, having had shorter intervals, were "faster" than the patterns in Experiment II, which had interstimulus intervals longer by $50 \%$ or more. It may be that rapidly flowing temporal information is processed more accurately when it all enters via one sense mode than when it enters via two sense modes and must be compared in an area of joint association; slower flowing information may be processed crossmodally with no loss of accuracy. Having interstimulus intervals lengthened may have made pattern speed go from a rate, in Experiment 1 , at which crossmodal transfer was impaired to a rate, in Experiment II, where such transfer was "in step." Thus, in comparing intramodal to crossmodal transfer at slow pattern speeds, no differences should occur. As pattern speed is increased, differences should not arise until a critical transition point is reached, beyond which crossmodal performance would decline faster than intramodal performance. This transition point may well have been touched or crossed in Experiment I.

A second procedural difference between experiments was that, in Experiment II, not only were the patterns slower but also the ratio of the long interval to the short interval was greater, yielding more correct responses in one intramodal combination, AA. Thus, it might be proposed that crossmodal matching is only inferior to intramodal matching for difficult to recognize patterns but not for easy to recognize patterns.

Pattern speed and item difficulty covaried in the two experiments, so that at first glance it is not possible to separate 
their effects. The results, however, indicated that the nine items varied in difficulty. If difficulty is responsible for the differences between crossmodal and intramodal performance and "errors per item" is as valid a measure of difficulty as is the ratio of the long to short interval, then the intramodal-crossmodal difference should increase directly with the increase in item difficulty, i.e., the slope of the function relating errors per item to order of difficulty should be steeper for crossmodal than for intramodal matching. Analysis of the data indicates that the crossmodal slope is $4 \%$ greater and $5 \%$ smaller than intramodal slope in Experiments I and II, respectively. These differences are insignificant, and, furthermore, the intramodal and crossmodal slopes resulting from combining the two experiments are equal. This does not exclude the possibility that the ratio of the long to short interval may affect the crossmodal-intramodal difference, but it does reduce one's confidence in its likelihood.

Anatomical support for differences in intramodal vs crossmodal transfer in vision and audition is cited in Geschwind (1965). He states that there are no direct connections in man from primary visual areas to primary auditory areas, but that there are some connections from auditory association areas to visual association areas, with less in the opposite direction. Thus, crossmodal processes do involve more complex associations than do intramodal ones.

\section{Patterns}

Analyzing the items according to order of presentation showed that the likelihood of an error was influenced by which pattern occurred first in an item. When Pattern 2 was first, significantly fewer errors occurred than when Patterns 1 and 3 were first. This superiority of Pattern 2 did not occur when items were analyzed according to which pattern came second. The sequence effect is strikingly illustrated by comparing errors for the item pair $(2-3,3-2)$, which contains Patterns 2 and 3 , but in opposite order. While Item 2-3 produced 14 errors, Item $3-2$ produced 66 errors.

One might have thought before this investigation that the sequence of presentation in a "same-different" pair comparison would have little effect on error rates. Rationally, however, one can distinguish different processes that may accompany the first and second patterns The first pattern must be received and stored; the second need only be received and compared to the pattern in storage. If Patterns 1, 2, and 3 differed in their capacity to be stored or retained, it would be reflected by differences in error rates for items beginning with those patterns. These differences in error rate did occur here. It does not necessarily imply that the patterns differed in storability, but one can find some basis for this conclusion from Garner's (1970) discussion.

The three patterns used here are, according to Garner, all included in a repeating single "basic" pattern, of which the first six elements are: $0 \times X 0 X X \ldots$ Using 0 for "long" and X for "short," Pattern 1 comprises elements 1-3 or 4-6, i.e., OXX; Pattern 2 is seen in Elements 3-5; Pattern 3 is in Elements 2-4. Although parts of the same "basic" pattern, the three patterns here differ in symmetry. Only Pattern 2 is symmetrical, sounding or looking the same backwards and forwards, i.e., X0X. Asymmetrical Patterns 1 and 3 belong in the same class, since Pattern 1 is Pattern 3 backwards, i.e., its own reflection. Symmetry is related to "goodness," which makes patterns easier to identify and remember, and, since Pattern 2 is the only symmetrical one used here, it would be expected (as was found here) that when it was first errors would be fewest; on the other hand, Patterns 1 and 3 , being "two of a kind" should be equally difficult. The present study did indeed reveal no significant differences between them when they were first in an item. The same argument regarding the influence of symmetry on errors may be applied to the analysis of items, according to which pattern was second. In this analysis, however, no differences in errors occurred between items ending in Patterns 1 and 3 vs Pattern 2. Since the only apparent difference in the processing of the first and second pattern of a comparison was that the first pattern had to be momentarily stored, it may be that "goodness" is more important to memory than to identification. In Garner's study, these two processes are not separable.

Whereas no significant differences were found between Patterns 1 and 3, it should be noted that similar trends occurred in both experiments, viz, Pattern 3 yielded more errors than did Pattern 1 when they were first, while the opposite was true when they were second. Thus, something in addition to "goodness" may influence pattern difliculty. Error analysis is an inefficient method of determining difficulty in the present experiments, since only $20 \%$ of the responses gathered are errors. In the future it would be valuable to collect response latencies as well. This additional information would clarify the role of symmetry and other factors in item difficulty, as well as provide some differentiation, if any exists, between items requiring a "same" judgment vs those requiring a "different" judgment.

\section{CONCLUSIONS}

The present study showed that in normal young adults: (1) Visual rhythmic patterns are more difficult to recognize and match than are comparable auditory patterns. (2) By equating, as much as possible, the difficulties of the visual and auditory rhythms, crossmodal matches are as easy as intramodal matches for patterns having no more than three stimuli per second, but are more difficult for patterns having as many as 4.5 stimuli per second. (3) Even in the small universe of patterns containing just four stimuli, there is consistency in the relative difficulty of items (patterns paired for comparison) between experiments, and among intramodal and crossmodal conditions. (4) A symmetrical pattern, when it is the first presented in a comparison, yields fewer errors than do asymmetrical patterns, but, when it is the second presented in a comparison, yields the same number of errors as do asymmetrical patterns.

Subsequent studies of pattern recognition should consider measures other than error frequency and expand the number of patterns evaluated in a systematic manner.

\section{REFERENCES}

BIRCH, H. G., \& BELMONT, L. Auditory-visual integration in normal and retarded readers. American Juurnal of Orthopsychiatry, 1964. $34,852-861$.

BIRCH, H. G., \& LEFFORD, A, Intersensory development in children. Monographs of the Society for Research in Child Development, 1963,28 , No. 89.

BRYANT, P. E. Comments on the design of developmental studies of crossmodal matching and crossmodal transfer. Cortex, 1968, 4, 127-137.

COLE, M.. CHOROVER, S. L., \& ETTLINGER, G. Crossmodal transfer in man. Nature, 1961, 191, 1225-1226.

ETTLINGER, G. Analysis of cross-modal effects and their relationship to language. In C. H. Millikan and F. L. Darley (Eds.), Brain mechanisms underlying speech and language. New York: Grune \& Stratton, 1967.

FRAISSE, P. Rythmes auditifs et rhthmes visuels. L'Année Psychologique, 1948, 49, $21-42$.

GARDNER, D. B. Children's perception of time and rhythm: Intersensory determinants. Paper read at Rocky Mountain Psychological Association meeting, 1968.

GARNER, W. R. Good patterns have few alternatives. American Scientist, 1970, 58, 3442.

GARNER, W. R., \& GOTTWALD, R. L. The perception and learning of temporal patterns. Quarterly Journal of Experimental Psychology, 1968, 20, 97-109. 
GAULT, R. H., \& GOODFELLOW, L. D. An empirical comparison of audition, vision, and touch in the discrimination of temporal patterns and ability to reproduce them. Journal of General Psychology, 1938, 18, 4147.

GESCHWIND, N. Disconnexion syndromes in animals and man. Part I. Brain, 1965, 88, 237-294.

MILLER, R. G. Simultaneous statistical inference. New York: McGraw-Hill, 1966.

MUEHL, S., \& KREMENAK, S. Ability to match information within and between auditory and visual sense modalities and subsequent reading achievement. Journal of Educational Psychology, 1966, 57, 230-239.

RUBINSTEIN, L. Intersensory and intrasensory effects in simple reaction time. Perceptual \& Motor Skills, 1964, 18, 159-172.

TAUBMAN, R. E. Studies in judged number: II.
The judgment of visual number. Journal of General Psychology, 1950, 43, 195-219.

THOR, D. H., \& HOLDEN, E. A. Visual perception of sequential numerosity by normals and retardates. Journal of Abnormal Psychology, 1964, 74, 676-682.

WHITE, C. T., \& LICHTENSTEIN, M. Some aspects of temporal discriminations. Perceptual \& Motor Skills, 1963, 17,471-482.

(Accepted for publication October 30, 1970.) 